\section{Discussion}

Laryngospasm as a solitary manifestation of epilepsy is rare. It was reported in a girl who had a long history of repeated seizures. ${ }^{3}$ Nocturnal dyspnoeic attacks in children were described by Jackson and Jackson' ${ }^{4}$ and defined as 'sudden dyspnoeic seizures' but no electroencephalographic correlates were reported.

The usual causes of laryngospasm, hypocalcaemia, hypoparathyroidism, or hypomagnesaemia are encountered infrequently and can easily be ruled out. Laryngospasm during anaesthesia is fairly common. The reflex laryngospasm which may appear during light anaesthesia is somewhat similar to our case. Both occur during sleep and are initiated by a remote stimulus which most probably reaches the larynx via the superior laryngeal nerve. The high frequency of attacks in our patient enabled easy documentation of the EEG abnormalities during normal sleep. In view of our findings, we feel that an EEG should be done in selected cases of recurrent nocturnal laryngospasm, especially those associated with alteration of consciousness.
The prompt therapeutic response to carbamazepine both in our patient and in another ${ }^{3}$ supports the suggestion that this is an 'epileptic equivalent', and renders other therapeutic means unnecessary.

We thank Perez Lavi for performing the polygraphic sleep study.

\section{References}

1 Walls T J, Newman P K, Cumming W J K. Recurrent apnoeic attacks as a manifestation of epilepsy. Postgrad MedJ 1981 ; 57: 575-6.

2 Nelson D A, Ray C D. Respiratory arrest from seizure discharges in limbic system. Arch Neurol 1968; 19: 199-207.

3 Ravindran M. Temporal lobe seizure presenting as 'laryngospasm'. Clin Electroencephalogr 1981; 12: 129-30.

4 Jackson C, Jackson C L. Diseases of the nose, throat, and ear. Philadelphia: Saunders, 1945: 459.

Correspondence to Dr J Amir, Department of Paediatrics, Beilinson Medical Centre, Petah Tiqva 49 151, Israel.

Received 20 September 1982

\title{
Recurrent meningitis secondary to concealed cerebrospinal fluid otorrhoea
}

\author{
KEVIN P MANNING AND ROGER GUDRÜN \\ Department of Otorhinolaryngology, Walton Hospital, Liverpool
}

SUMMARY Three children who suffered recurrent attacks of meningitis which were eventually found to be due to a concealed cerebrospinal fluid otorrhoea are presented. Between them they had 12 attacks of meningitis before a firm diagnosis was made and treatment undertaken to localise and close the subarachnoid middle ear fistula.

Meningitis nowadays is associated with an overall mortality of $8 \%{ }^{1}$ Streptococcus pneumoniae is the organism generally associated with recurrent meningitis. From this the mortality rate varies from $13 \%$ in the UK and the Republic of Ireland ${ }^{1}$ to $51 \%$ in Nigeria. ${ }^{2}$ Complications-such as auditory and visual deterioration and hydrocephalus-are common and occur in $21 \%$ of cases. ${ }^{3}$ Every effort should be made to find the cause in any patient with recurrent meningitis.

\section{Case reports}

Case 1. A 15-year-old girl was seen with cerebrospinal fluid (CSF) rhinorrhoea. She had had rightsided blindness and ophthalmoplegia since a head injury at age 5 years. She had had meningism and an extradural abscess secondary to acute mastoiditis 4 years previously. Subsequently she had 2 attacks of meningitis preceded by CSF rhinorrhoea.

Otoscopic examination of the tympanic membrane showed a fluid level suggesting she had CSF otorrhoea. Exploration of the mastoid confirmed this. The fluid came from a defect in the roof of the mastoid, at the site of the previous extradural abscess. This was repaired from below with temporalis fascia and a free muscle graft.

Case 2. This 14-year-old boy had 5 attacks of pneumococcal meningitis. At age 8 years he had 
fractured his right temporal bone. Two craniotomies with a fascial repair of the fracture had failed to stop these attacks.

Tomograms of the temporal bone showed a hairline fracture extending into the vestibule of the inner ear.

Exploration of the middle ear showed a fractured stapes footplate, manipulation of which resulted in a profuse flow of CSF. The leakage was controlled by removing the footplate fragments and packing the inner ear with a free muscle graft through the oval window.

Case 3. A 6-year-old girl was seen with a profuse right-sided CSF otorrhoea after a myringotomy. She had a profound bilateral sensorineural deafness. Before this she had had 4 attacks of pneumococcal meningitis, the first occurring at age 20 months. Her deafness was noticed by her mother after the first attack of meningitis.

Tomograms of the inner ear showed an abnormally dilated vestibule and lateral semicircular canal and a cyst-like cochlea (Figure).
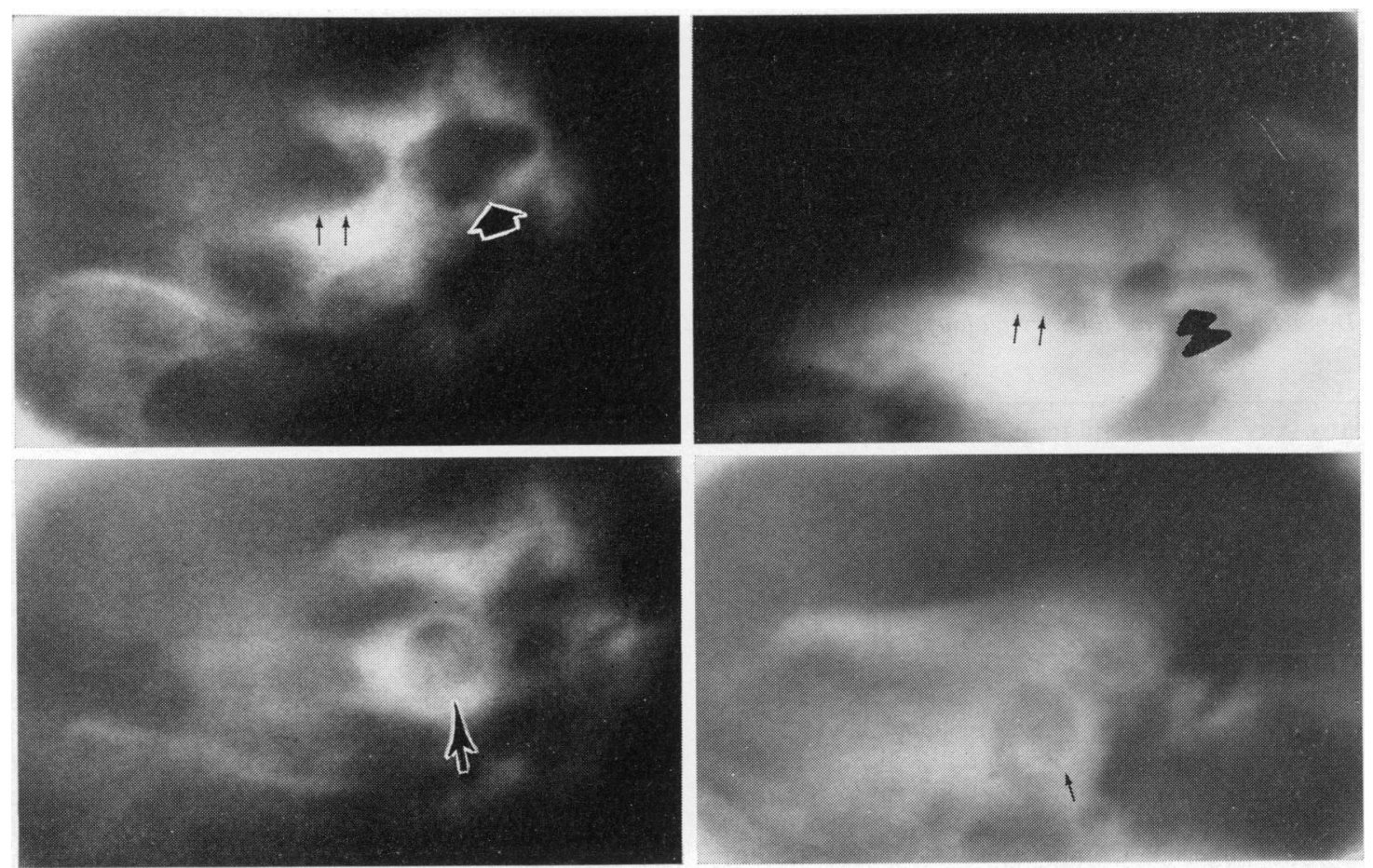

Figure Tomograms of the inner ear showing (top left) abnormally dilated vestibule and lateral semicircular canal and (bottom left) the cyst-like cochlea. For comparison (top right) is shown a normal vestibule and lateral semicircular canal (large arrow) and (bottom right) a normal cochlea showing the spiral modiolus. Parallel arrows show the internal auditory meatus.

Exploration of the middle ear showed a leakage of fluid from the oval window. Removal of the stapes was followed by a profuse flow of CSF. This was stopped by packing a free muscle graft into the vestibule of the inner ear through the oval window.

\section{Discussion}

These 3 patients demonstrate a rare cause of recurrent meningitis. The pathway of infection was by a subarachnoid middle ear cleft fistula in each. The fistula resulted from a different disease in each patient: inflammatory, traumatic, and congenital.

In Case 1 the middle ear was overlooked because the leakage presented each time as CSF rhinorrhoea. Examination of the tympanic membrane at a time of active rhinorrhoea and the presence of fluid led to a correct diagnosis as to the site of the fistula.

In Case 2 intracranial repair of the fracture site with fascia failed to control the attacks of meningitis, probably because the fracture also extended to the posterior surface of the temporal bone and from there into the inner ear. 
Case 3 is a rare congenital abnormality of the inner ear which usually presents as recurrent meningitis. This abnormality is now well documented. ${ }^{4}$ It should be suspected as a cause of recurrent meningitis in a child who has a sensorineural deafness. The presence of fluid behind the tympanic membrane should make one suspicious and the diagnosis can be confirmed by tomograms of the inner ear. The CSF fistula is from the fundus of the internal auditory canal into the vestibule of the inner ear and from there into the middle ear through a defect in the stapes footplate. The simplest and safest way to close this defect is through the middle ear.

A fluid level behind the tympanic membrane is usually due to secretory otitis media. In patients with meningitis or CSF rhinorrhoea, the possibility that the fluid in the middle ear may be CSF should be considered.
We thank our neurosurgical colleagues for referring these cases.

\section{References}

1 Anonymous. Deaths from bacterial meningitis. $\mathrm{Br} \mathrm{Med} \mathrm{J}$ 1973 ; i: 623.

2 Baird D R, Whittle H C, Greenwood B M. Mortality from pneumococcal meningitis. Lancet 1976; ii: 1344-6.

${ }^{3}$ Spink W W, Su C K. Persistent menace of pneumococcal meningitis. JAMA 1960; 173: 1545-8.

4 Parisier S C, Birken E A. Recurrent meningitis secondary to idiopathic oval window CSF leak. Laryngoscope 1976; 86: $1503-15$.

Correspondence to K P Manning FRCS, Walton Hospital, Rice Lane, Liverpool L9 1AE.

Received 8 September 1982

\section{The Rottenrow endotracheal tube holder}

\author{
ALISON M KERR \\ Royal Hospital for Sick Children, Glasgow
}

SUMMARY A new device is described which holds the endotracheal tube securely in the mouth of the neonate during ventilation.

Several devices have been produced, but displacement of the endotracheal tube remains one of the most common problems in neonatal nurseries. Despite its obvious disadvantages many units still prefer the security obtained by driving a large safety pin through the tiny endotracheal tube as it crosses the infant's mouth.

A device should possess the following features: (1) The grip should remain secure without occlusion of the endotracheal tube in the warm, mucusy mouth of the infant. (2) It should be able to be fixed simply from the side of the tube and allow adjustment without having to be detached from the ventilating machine. (3) It should be suitable for all sizes and types of tube used in the newborn. (4) Small loose parts which could be swallowed or inhaled should be absent. (5) It should invade the mouth as little as possible and allow oral toilet. (6) The material should be pliable so as not to damage the skin. (7) There should be a choice of fixation-tapes or adhesive. (8) It should be cheap and capable of reuse.
With these in mind a device has been designed in flexible polyvinylchloride (PVC). It has been tested and modified in use, until a shape has been arrived at which suits the nature of the material, the manufacturing process, and the clinical requirements.

\section{Nature and use of the 'Rottenrow device'}

The device is made of PVC. The endotracheal tube strap is perforated at $2 \mathrm{~mm}$ intervals by $2 \mathrm{~mm}$ holes. The stainless steel peg is two-thirds embedded in the PVC pillar (Fig. 1).

\section{Instructions}

The endotracheal tube is introduced into the infant's airway in the conventional manner. While it is supported in position by the hand in the angle of the mouth, the device, with the endotracheal tube strap open, is brought up from the side and placed so that the tube lies snugly in its groove and the narrow part of the face strap is closely applied to the upper lip. The endotracheal tube strap is then stretched round the tube and fixed with moderate tension to the steel peg. The tube must then be checked to make sure it is held firmly without occlusion. The face strap may be secured directly to 